\title{
The Evolution of the Stress Concept
}

The originator of the concept traces its development from the discovery in 1936 of the alarm reaction to modern therapeutic applications of syntoxic and catatoxic hormones

Everybody knows what stress is and nobody knows what it is. The word stress, like success, failure, or happiness, means different things to different people and, except for a few specialized scientists, no one has really tried to define it although it has become part of our daily vocabulary. Is it effort, fatigue, pain, fear, the need for concentration, the humiliation of censure, loss of blood, or even an unexpected success that requires complete reformulation of one's life? The answer is yes and no. That is what makes the definition of stress so difficult. Every one of these conditions can produce stress, and yet none of them, can be singled out as being "it" since the word applies equally to all others as well.

Yet, how are we to cope with the stress of life if we cannot even define it? The businessman who is under constant pressure from his clients and employees alike, the air traffic controller who knows that a moment of distraction may mean death to hundreds of people, the athlete who desperately wants to win a race, and the husband who helplessly watches his wife slowly and painfully die of cancer-all suffer from stress. The problems they face are totally different, but medical research has shown that in many respects their bodies respond in a stereotyped manner with identical biochemical changes, meant fundamentally to cope with any type of increased demand upon the human machinery. The stress-producing factors-technically called stressors-are different, and yet they all produce essentially the same biologic stress response. This distinction between stressor and stress was perhaps the first important step in the scientific analysis of that most common biologic phenomenon that we all know only too well from personal experience.

But if we want to use what the laboratory has taught us about stress in formulating our own philosophy of life, if we want to avoid its bad effects and yet be able to enjoy the pleasures of accomplishment, we have to learn more about the nature and mechanism of stress. To succeed in this, we must concentrate on the fundamental technical data which the laboratory has given us as a basis for a scientific philosophy of conduct. Examination of the data seems to be the only way of finding purpose in life without having to fall back upon traditional beliefs whose acceptance depends primarily on indoctrination (1).

\section{What is stress?}

Stress is the nonspecific response of the body to any demand made upon it. In order to understand this definition we must first comprehend what is meant by "nonspecific." Each demand made upon our body is in a sense unique, that is, specific. When exposed to cold we shiver to produce more heat, and the blood vessels in our skin contract to diminish loss of heat from the body surface. When exposed to heat we sweat, because evaporation of perspiration from he surface of our skin has a cooling effect. When we eat so much sugar that the blood-sugar level rises above normal, we excrete some of it and 
try to activate chemical reactions which will enable us to store or burn up the rest so that the blood sugar may return to normal. A great muscular effort, such as running up many flights of stairs at full speed, makes increased demands upon our musculature and cardiovascular system: the muscles will need more energy to perform this unusual work; hence the heart will beat more rapidly and strongly, and the blood pressure will rise to accelerate delivery of blood to the musculature.

Each drug and hormone has such specific actions: diuretics increase urine production; adrenalin augments the pulse rate and blood pressure, simultaneously increasing blood sugar, whereas insulin decreases blood sugar. Yet, no matter what kind of derangement is produced, all these agents have one thing in common: they also make an increased demand upon the body to readjust itself'. This demand is nonspecific; it requires adaptation to a problem, regardless of what that problem may be. That is to say, in addition to their specific actions, all agents to which we are exposed produce a nonspecific increase in the need to perform certain adaptive functions and then to reestablish normalcy, which is independent of the specific activity that caused the rise in requirements. This nonspecific demand for activity as such is the essence of stress.

From the point of view of its stress-producing, or stressor, activity, it is even immaterial whether the agent or situation we face is pleasant or unpleasant; all that counts is the intensity of the demand for readjustment or adaptation. The mother who is suddenly told that her only son died in battle suffers a terrible mental shock; if years later it turns out that the news was false, and the son unexpectedly walks into her room alive and well, she experiences extreme joy. The specific results of the two events, sorrow and joy, are completely different, in fact, opposite to each other; yet their stressor effect-the nonspecific demand to readjust to an entirely new situation-may be the same.

It is difficult to see how such essentially different things as cold, heat, drugs, hormones, sorrow, and joy could provoke an identical biochemical reaction in the organism. Yet this is the case; it can now be demonstrated by highly objective quantitative biochemical determinations that certain reactions of the body are totally nonspecific and common to all types of exposure.

It has taken medicine a long time to accept the existence of a stereotyped response to any kind of demand. It did not seem logical that different tasks, in fact any task, should cause the same response. And yet there are many analogies in everyday life where highly specific things or events share the same nonspecific feature. Take, for example, a house in which there are heaters, refrigerators, bells, and light bulbs which respectively produce heat, cold, sound and light in a most specific manner, they to function they depend upon just one common factor electricity. A member of a primitive tribe who had never heard of electricity would find it very difficult to accept that these manifold phenomena depend upon the satisfaction of a common demand-the provision of electric energy.

\section{What stress is not}


Since the term stress has been used quite loosely, many confusing and often contradictory definitions have been formulated; hence, it will be useful to add a few remarks stating clearly what it is not. Stress is not simply nervous tension; stress reactions do occur in lower animals, which have no nervous system, and even in plants. Stress is not the nonspecific result of damage. We have seen that it is immaterial whether an agent is pleasant or unpleasant; its stressor effect depends merely on the intensity of the demand made upon the adaptive work of the body. As I have explained elsewhere (2), "normal activities-a game of tennis or even a passionate kiss-can produce considerable stress without causing conspicuous damage."

Stress is not something to be avoided. In fact, it is evident from the definition given earlier that it cannot be avoided; no matter what you do or what happens to you, there arises a demand to provide the necessary energy to perform the tasks required to maintain life and to resist and adapt to the changing external influences. Even while fully relaxed and asleep, you are under some stress: your heart must continue to pump blood, your intestines to digest last night's dinner, your muscles to move your chest to permit respiration; even your brain is not at complete rest while you are dreaming.

Complete freedom from stress is death. Contrary to public opinion, we must not-and indeed cannot avoid stress, but we can meet it efficiently and enjoy it by learning more about its mechanism and adjusting our philosophy of' life accordingly (1).

\section{Historic development}

The concept of' stress is very old; it must have occurred even to prehistoric man that the loss of vigor and feeling of exhaustion that overcame him after hard labor, prolonged exposure to cold or heat, loss of blood, agonizing fear, or any kind of disease had something in common. He may not have been consciously aware of this similarity in his response to anything that was just too much for him, but when the feeling came he must have realized that he had exceeded the limits of what he could reasonably handle, in other words that "he had had it"

Man soon must have discovered also that whenever faced with a prolonged and unaccustomed strenuous task-be it swimming in cold water, lifting rocks, or going without food-he passes through three stages: at first the experience is a hardship, then one gets used to it, and finally one cannot stand it any longer. He did not think of this three-phase response as a general law regulating the behavior of living beings faced with an exacting task. The immediate necessities of finding food and shelter kept him too busy to worry about such concepts as the maintenance of the milieu interieur, homeostasis, $\alpha$ biologic stress. Yet, the vague outlines of all this were there, ready to be analyzed and translated from intuitive feelings into the precise terms of science, a language that can be appraised by intellect and tested by the critique of reason.

How could different agents produce the same result? Is there a nonspecific adaptive reaction to change as such? In 1926, as a second-year medical student, I first came across this problem of a stereotyped response to any exacting task. I began to wonder why 
patients suffering from the most diverse diseases have so many signs and symptoms in common. Whether a man suffers from severe loss of blood, an infectious disease, or advanced cancer, he loses his appetite, his muscular strength, and his ambition to accomplish anything; usually the patient also loses weight, and even his facial expression betrays that he is ill. What is the scientific basis of what I thought of at the time as the "syndrome of just being sick"? Could the mechanism of this syndrome be analyzed by modern scientific techniques? Could it be reduced to its elements and expressed in the precise terms of biochemistry, biophysics, and morphology? Could this reaction be subjected to scientific analysis?

It was not until 1936 that the problem presented itself again, now under conditions more suited to analysis. At that time, I was working in the Biochemistry Department of McGill University, trying to find a new hormone in extracts of cattle ovaries. I injected the extracts into rats to see if their organs would show unpredictable changes that could not be attributed to any known hormone. Much to my satisfaction, the first and most impure extracts changed the rats in three ways: (1) the adrenal cortex came enlarged, (2) the thymus spleen, lymph nodes, and all other lymphatic structures shrank, and (3) deep, bleeding ulcers appeared in the stomach and in the upper gut. Because the three types of change were closely interdependent they formed a definite syndrome. The changes varied from slight to pronounced, depending on the amount of extract I injected.

At first, I ascribed all these changes to a new sex hormone in the extract. But soon I found that all toxic substances-extracts of kidney, spleen, or even a toxin not derived from living tissue-produced the same syndrome. Gradually, my classroom concept of the "syndrome of just being sick" came back to me. I realized that the reaction I had produced with $\mathrm{m}$ impure extracts and toxic drugs was an experimental replica of this syndrome. Adrenal enlargement, gastrointestinal ulcers, and thymicolymphatic shrinkage were the omnipresent signs of damage to the body when under disease attack. The three changes thus became the objective indexes of stress and the basis for the development of the entire stress concept.

The reaction was first described in ad Nature (4 July 1936) as "A Syndrome Produced by Various Nocuous Agents" and, subsequently, it became known as the General Adaptation Syndrome (GAS) or biologic stress syndrome. In the same paper, I also suggested the name alarm reaction for the initial response, arguing that it probably represents the somatic expression of a generalized "call to arms" of the body's defensive forces.

\section{The general adaptatior syndrome}

The alarm reaction, however, was evidently not the entire response. Upon continued exposure to any noxious agent capable of eliciting this reaction, a stage of adaptation or resistance ensues. In other words, no organism can be maintained continuously in a state 
of alarm. If the agent is so drastic that continued exposure becomes incompatible with life, the animal dies during the alarm reaction within the first hours or days. If it can survive, this initial reaction is necessarily followed by the "stage of resistance." The manifestations of this second phase are quite different from-in many instances, the exact opposite of-those which characterize the alarm reaction. For example, during the alarm reaction, he cells of the adrenal cortex discharge their secretory granules into the bloodstream and thus become depleted of corticoid -containing lipid-storage-re material; in the stage of resistance, on the other hand, the cortex becomes particularly rich in secretory granules. Whereas in the alarm reaction, there is hemoconcentration, hypochloremia, and general tissue catabolism, during the stage of resistance, there is hemodilution, hyperchloremia, and anabolism, with a return toward normal body weight.

Curiously, after still more exposure to the noxious agent, the acquired adaptation is lost again. The animal enters into a third phase, the "stage of exhaustion," which inexorably follows as long as the stressor is severe enough and applied for a sufficient length of time. Because of its great practical importance, it should be pointed out that the triphasic nature of the GAS gave us the first indication that the body's adaptability, or "adaptation energy," is finite since, under constant stress, exhaustion eventually ensues. We still do not know precisely what is lost, except that it is not merely caloric energy, since food intake is normal during the stage of resistance. Hence, one would think that once adaptation has occurred and ample energy is available, resistance should go on Indefinitely. But just as any inanimate machine gradually wears out, so does the human machine sooner or later become the victim of constant wear and tear. These three stages are reminiscent of childhood (with its characteristic low resistance and excessive responses to any kind of stimulus), adulthood (during which the body has adapted to most commonly encountered agents and resistance is increased), and senility (characterized by loss Of adaptability and eventual exhaustion).

Since 1936, numerous additional biochemical and structural changes of Previously unknown origin have been traced to nonspecific stress. Among these, clinicians have given special attention to changes in the chemical constituents of the body and to nervous reactions. Much progress has also been made in the analysis of the hormonal mediation of stress reactions. It is now generally recognized that the emergency discharge of adrenalin represents only one aspect of the acute phase of the alarm reaction to stressors. The hypothalamus-hypophysisadrenocortical axis is at least equally important for homeostasis, and it probably participates in the regulation of many disease phenomena as well.

A vital part of the defense mechanism is the excitation of the hypothalamus (a nerve center at the base of the brain), which releases a chemical messenger that causes the hypophysis (a small gland just below the hypothalamus) to increase secretion of ACTH, the so called adrenocorticotrophic hormone, which in turn stimulates the adrenal cortex to produce corticoids. Most important among the corticoids are the glucocorticoids, such as cortisone, which cause thymus atrophy and influence glucose and, in general, organic metabolism. 
Various derangements in the secretion of these hormones can lead to maladies which I called "diseases of adaptation," because they are not directly due to any particular pathogen but to a faulty adaptive response to the stress induced by some pathogen. In this sense, many ailments, such as various emotional disturbances, mildly annoying headaches, insomnia, upset stomachs, sinus attack, crippling high blood pressure, gastric and duo- ulcers, certain types of rheumatic or allergic afflictions, as well as cardiovascular and kidney diseases appear essentially to be initiated or encouraged by the body itself because of its faulty adaptive reactions to potentially injurious agents.

The history of the GAS suggests that the key to real progress was the discovery of objective indexes of stress-adrenal enlargement, thymicolymphatic atrophy, and acute gastrointestinal ulcers. Yet even these signs were known to some physicians long before we began to realize that there is such a thing as a nonspecific stress syndrome. As early as 1842, in England, Curling described acute gastrointestinal ulcers in patients who suffered extensive skin burns. In 1867, the German surgeon Billroth reported similar findings after major surgical interventions complicated by infections. But there was no reason to connect these lesions with other changes that today would be regarded as part of the stress syndrome for example, with those described at the Pasteur Institute in Paris by Roux and Yersin, who noted that the adrenals of guinea pigs infected with diphtheria are often enlarged, bloodshot, and hemorrhagic. In fact, these people did not even know about each other's work.

The so-called accidental thymus atrophy and the loss of body weight of individuals affected by disease have been described so often that it would be difficult to trace their history; but who would have thought of them in relation to what the American physiologist W. B. Cannon described as "emergency adrenalin secretion" in response to fear or rage? One important link was still missing-that connecting all these scattered observations as merely individual manifestations of a single coordinated syndrome.

\section{A single stereotyped response}

There remained two apparently insurmountable obstacles to formulating the concept of a single stereotyped response to stress:

1. Qualitatively different agents of equal toxicity (or stressor potency) do not necessarily elicit exactly the same syndrome in different people.

2. Even the same degree of stress, induced by the same agent, may produce different lesions in different individuals.

It took many years to show that qualitatively different agents differ only in their specific actions while their nonspecific stressor effects (such as ACTH- or corticoid-secretion and thymicolymphatic involution) are essentially the same, unless these happen to be modified by the superimposed specific effects of the evocative agents. 
On the other hand, the fact that even the same stressor can cause different lesions in different individuals has been traced to "conditioning factors" that can selective, y enhance or inhibit one or the other stress effect. This conditioning may be internal (genetic predisposition age, or sex, for example) or external (treatment with certain hormones drugs, or dietary factors) (see pig' 3). Under the influence of such conditioning factors, a normally well tolerated degree of stress can become pathogenic and cause diseases of adaptation which affect predisposed areas of the body selectively.

The concept that every specific disease entity must have its own particular cause gained acceptance mainly during the nineteenth century. Following the emergence of modern bacteriology, it became evident that the characteristic syndrome of any one infectious malady, such as tuberculosis or diphtheria, could be elicited only by its own specific germ. Soon afterward, research in the field of nutrition and endocrinology similarly showed that one kind of derangement can always be traced to the lack or the excess of a particular vitamin or hormone.

Very few diseases are monocausal in the sense that their development is the inevitable consequence of one particular pathogen (e.g. paralysis after spinal cord transection or sterility following ovariectomy). Many maladies are predominantly pluricausal in that the "soil factor" (individual variations of disease proneness) plays an important role: influenza does not befall all persons exposed to influenza virus, but only the susceptible ones. The typical pluricausal diseases are not caused by any particular pathogen but are the consequence of "pathogenic constellations." To this group belong peptic ulcers, accidental thymus involution, many forms of collagen disease, nephrosclerosis, thrombohemorrhagic lesions, atopic dermatitis, various neuroses, and many others. In fact, the possibility of a pluricausal pathogenesis must be considered in the case of all idiopathic maladies.

Several experimental models of pluricausal diseases (e.g. electrolyte -steroidcardiopathy, calciphylaxis, calcergy, progeria-like syndrome, the thrombohemorrhagic phenomenon, acute conditioned necrosis, anaphylactoid edema) have been developed at the Institute of Experimental Medicine and Surgery at the University of' Montreal, and comparative studies were made from the pathologic and pharmacologic points of view. These models have in common that they can be produced by several combinations of at least two, in themselves inactive. Essentially different pathogens (1) "sensitizers" which induce a latent predisposition for a specific reaction form (e.g. inflammation, necrosis, calcification, thrombosis, or hemorrhage) and (2) "challengers which unmask this predisposition by making the disease manifest and determining its localization.

An example of one of these experimental disease models is the electrolyte -steroid -cardiopathy (ESC). Many structurally distinct, experimental cardiopathies can be produced or prevented at will by varying combinations of electrolytes, steroids, and stress. As a rule, electrolytes, corticoids, or stressors produce no consistent cardiac changes by themselves; only certain combinations of them are cardiotoxic. The different types (characterized by hyalinosis $=\mathrm{ESCH}$, or by calcification = ESCC) are described at 
length in two monographs $(4,5)$. Here, I shall deal only with the ESCN (the electrolyte -steroid-cardiopathy with necrosis) and its prevention by chemical means.

The ESCN is characterized by large infarctoid necroses that develop in the absence of coronary occlusion as a result of the combined action of certain steroids and sodium salts, stress, or excessive lipid ingestion. For the routine screening of Potentially prophylactic agents, we usually employ variants of these models produced by glucomineralocortcoids (e.g. methylchlorocortisol) in combination with sodium Phosphate, stressors, or lipids. These models of metabolic myocardial necrosis have been selected because unlike the cardiopathies elicited by toxic drugs (plasmocid, papain, etc.), they depend upon factors likely to play a role also in cardiac diseases of man.

The cardiac lesions thus produced are associated with a severe drop in myocardial potassium and an increase in sodium. It is of great sgnificance that stress plays a decisive role in the ESCN since, in man, physical and mental exertions have long been suspected of provoking myocardial infarction. It has been found that cardiac necroses of the ESCN can be prevented in animals by oral administration of KCI or $\mathrm{MgC1} 2$ (see Fig. 4), and industry has made available a large number of potassium and magnesium preparations, among them organic salts, recommended for the prophylaxis of myocardial infarction and even for treatment during the postinfarction period.

\section{Syntoxic and catatoxic responses}

It was the great French physiologist Claude Bernard who, during the second half of the nineteenth century-well before anyone thought of stress-first pointed out clearly that the internal medium of living organisms is not merely a vehicle for carrying nourishment to cells but that "it is a fixity of the milieu interieur which is the condition of free and independent life." Some fifty years later, Cannon suggested the designation homeostasis for "the coordinated physiological processes which maintain most of the steady states in the organism"-the ability to stay the same or static from the Greek homoios = similar and stasis $=$ position, standing.

What is meant by the "fixity of the milieu interieur"? Everything inside the skin and even the, skin itself constitutes the internal medium. In order to maintain a healthy life, nothing within the body must be allowed to deviate far from the norm; if something does, the individual will become sick or even die. There are many complex biochemical mechanisms which ensure the fixity, the steadiness, of the milieu interieur-these coordinated stabilizing responses are called homeostatic. Let us look briefly at how they work.

The biochemical analysis of the stress syndrome showed that homeostasis depends mainly upon two types of reactions -syntoxic (from syn = together) and catatoxic (from cata = against). Apparently, in order to resist different stressors, the organism can regulate its own reaction through chemical messengers and nervous stimuli which either pacify or incite to fight. Syntoxic stimuli act as tissue tranquilizers, creating a state of passive tolerance, which permits a kind of symbiosis or peaceful coexistence with 
aggressors. The catatoxic agents cause chemical changes (mainly through the production of destructive enzymes), which lead to an active attack upon the pathogen, usually by accelerating its metabolic degradation.

Presumably, in the course of evolution, the body has learned to defend itself against all kinds of aggressors (whether arising in the body or externally introduced) through two basic mechanisms, which help us put up with the aggressor (syntoxic) or destroy it (catatoxic). Among the most effective syntoxic hormones are substances produced by the adrenal cortex. I have called these corticoids. The best known members of this group are the anti-inflammatory corticoids such as cortisone and related substances which inhibit inflammation and many essentially defensive immune reactions. These corticoids are being effectively used in the treatment of diseases in which inflammation itself is the -major cause of trouble (inflammation of the joints, eyes, or respiratory passages). Likewise, they have a marked inhibitory effect on the active rejection of grafted foreign tissues (e.g. hearts or kidneys).

It is not immediately evident why it should be advantageous to inhibit inflamation or interfere with the rejection of foreign tissues, since both phenomena are essentially useful defense reactions. The main purpose of inflammation is to localize irritants (for example, microbes) by putting a barricade of inflammatory tissue around them to prevent their spread into the blood, which could lead to blood poisoning and death. The suppression of this basic defense reaction is an advantage, however, when a foreign agent is in itself innocuous and causes trouble only by inciting inflammation. In such cases, inflammation itself is what we experience as a disease. Thus, in many patients who suffer from hay fever or extreme inflammatory swelling after an insect sting, suppression of defensive inflammation is essentially a cure, because the invading stressor agent is not in itself dangerous or likely to spread and kill. In the case of grafts, it may even be lifesaving.

At this point, it is useful to distinguish between direct and indirect pathogens. Direct pathogens cause disease regardless of the body's reaction, whereas indirect pathogens cause damage only because they provoke exaggerated defensive responses. If a man accidentally exposes his hand to a strong acid, alkali, or boiling water, damage will occur regardless of his reactions, because all these are direct pathogens, which would cause damage even to the hand of a dead man, who obviously could not put up any vital defense reactions. Most of the common inflammatory irritants, including allergens, however, are essentially indirect pathogens: they cause disease only through exaggerated and purposeless defense reactions which they stimulate.

During evolution, immunologic reactions which lead to destruction of microbes, grafts, and other foreign tissues undoubtedly developed as useful defensive mechanisms against potentially dangerous foreign materials. However, when-as in the case of many allergens, heart transplants, etc.-the attack against the "foreign" agent is unnecessary or even harmful, man can improve upon the wisdom of Nature by suppressing this hostility.

On the contrary, when the aggressor is dangerous, the defensive reaction should not be suppressed but, if possible, increased above the normal level, which can be done, for 
example, by catatoxic substances that carry the chemical message to the tissues to destroy the invaders even more actively than would normally be the case.

An example from daily life will illustrate in principle how diseases can be produced indirectly by our own inappropriate or excessive adaptive reactions. If you meet a helpless drunk who showers you with insults but is obviously quite unable to do you any harm, nothing will happen if you take a "syntoxic attitude"-go past and ignore him. However, if you respond catatoxically and fight, or even only prepare to fight, the result may be tragic. You will discharge adrenalin-type hormones that increase blood pressure and pulse rate, while your whole nervous system will become alarmed and tense in anticipation of combat, If you happen to be a coronary candidate, the result may be a fatal brain hemorrhage or cardiac accident. In this case, who is the murderer? The drunk didn't even touch you. This is biologic suicide! Death is caused by choosing the wrong reaction.

If, on the other hand, the man who showers you with insults is a homicidal maniac with a dagger in his hand, evidently determined to kill you, it is essential to take an aggressive catatoxic attitude. You must try to disarm him even at the risk of injury to yourself from the physical accompaniments of the alarm reaction in preparation for combat. It is clear that , contrary to common opinion, Nature does not always know best because, both on the cellular and interpersonal level, we do not always recognize what is , lid what is not worth fighting.

These are the considerations that led me to formulate my personal philosophy of conduct in the terms of this simple jingle:

Fight for the highest attainable aim

But do not put up resistance in vain.

\section{Adaptation to stress}

When faced with stressful situations that require systemic adaptation, the organism can respond through three essentially distinct mechanisms: nervous, immunologic and phagocytic, and hormonal. The first includes conscious planning of defense, innate or conditioned reflexes, and autonomic "emergency reactions" (partly mediated through neurohormones). The immunologic and phagocytic mechanism involves antibody formation and, activation of the recticuloendotlielial system. The hormonal mechanism works through syntoxic hormones that permit tolerance of the pathogen without attacking it (e.g. glucocorticoids that prevent inflammation without destroying its cause) or catatoxic substances that eliminate the aggressor (e.g. certain steroids and drugs that accelerate the biodegradation of toxicarts without inducing tissue resistance to them) (7).

The new concept of heterostasis (8) -homeostasis artificially raised to a superior level -includes both syntoxic and catatoxic reactions induced by natural or artificial exogenous compounds. It excludes all -therapeutic measures that act direc-.1y, or "passively" -i.e. not by, stimulating the body's own adaptive capacities. Such direct, or passive, measures 
include for example: (1) antidotes that act directly upon Potential pathogens (e.g. buffers that neutralize acids, chemicals that inactivate poisons by directly combining with them, antimicrobial agents); (2) all forms of substitution therapy that merely represent chemical or mechanical prostheses (e.g. vitamin C for scurvy, corticoids for Addison's disease, -transplanted or artificial kidneys for renal failure); (3) ablation of diseased tissues such a tumors; (4) passive "shielding" (e.g. lead screens against X rays, receptor blockade against drugs, competition for a substrate).

The types of adaptation that depend upon active participation of the body are homeostatic (both syntoxic and catatoxic) or heterostatic (again, both syntoxic and catatoxic) (8). Such adaptation comprises all active defense reactions regardless of their nervous, 3 . immunologic, phagocytic, or hormonal mediation. The list incorporates a general classification of adaptive mechanisms, including both those in which the milieu interieur participates actively or passively (see Fig. 5).

"Adaptability is probably the most distinctive characteristic of life. In maintaining the independence and individuality of natural units, none of the great forces of inanimate matter are as successful as that alertness and adaptability to change which we designate

as life and the loss of which is death. Indeed, there is perhaps even a certain parallelism between the degree of aliveness and the extent of adaptability in every animal-in every man"-Selye (9).

\section{References}

1. H. Selye. In preparation. Stress without Distress. New York, Philadelphia: Lippincott.

2.H. Selye. 1956. The Stress of Life. New

York: McGraw-Hill.

3.H. Selye. 1952. The Story of the Adaptation Syndrome. Montreal: Acta Inc., Med. Publ.

4.H. Selye. 1958. The Chemical Preuention of Cardiac Necroses. New York: Ronald Press.

5. H. Selye, 1961. The Pluricausal Cardiopathies. Springfield: Charles C Thomas.

6. H. Selye and R. K. Mishra. 1958. Prevention of the " phosphate - steroidcardiopathy" by various electrolytes. Amer. Heart J 55:163.

7.H. Selye. 1971. Hormones and

Resistance. Heidelberg: Springer-Verlag.

8. H. Selye. 1973. Homeostasis and heterostasis. Perspect. Biol. Med. 16:441.

9.H. Selye. 1950. Stress. Montreal: Acta

Inc., Med. Publ. 